\title{
AVALIAÇĀO CLINICO-QUANTITATIVA DA AÇÃO DO CIBA 34.647-Ba SOBRE A ESPASTICIDADE
}

\author{
ISRAEL ROITMAN * \\ ABRAO ANGHINAH **
}

Embora a espasticidade constitua deficiência funcional bastante acentuada em numerosos doentes, bem poucos medicamentos se mostraram eficazes no seu tratamento.

Trabalhos recentes $3,6,7,10,12,13$ mostraram a influência favorável do Ciba 34.647-Ba, um derivado do ácido gama-amino-butírico (GABA), do qual se conhecem propriedades inibidoras ao nivel do sistema nervoso. central. $O$ GABA não atravessa a barreira hemato-encefálica, porém o ácido beta-(4-clorofenil)-gama-amminobutírico, quando administrado por via oral, transpõe facilmente essa barreira ${ }^{14}$.

Observações em animais de laboratório 8,18 revelaram que a droga deprime a atividade reflexa monossináptica espinal tanto em animais descerebrados (seja a descerebração de origem cirúrgica ou anêmica) como em animais espinais. As aferências sensitivas dos fusos musculares desaferentados e a transmissão neuromuscular não sofrem qualquer tipo de alteração. Estas observações pe:mitem supor que o local de ação da droga seja na medula espinal, ao nível das fibras aferentes provenientes dos fusos musculares. A inibição seria présináptica, isto é, antes da sinapse com os motoneurônios alfa. Por outro lado a droga diminui a atividade tônica dos motoneurônios gama, embora sua ação sobre a atividade fásica não tenha sindo ainda suficientemente investigada.

Estudos mais recentes $\mathbf{2 , 4 , 8 , 1 3 , 1 5}$, utilizando métodos e critérios os mais diversos, procuram demonstrar a ação do Ciba 34.647-BA sobre a espasticidade resultante de lesões em diferentes niveis do sistema nervoso central.

No presente estudo utilizamos a quantificação clínica da sintomatologia neurológica para a análise da ação deste derivado do GABA em pacientes com incapacidades motoras devidas a lesões da medula espinal, às quais se associou componente espástico importante.

\section{CASUISTICA E METODOS}

Os dados de identificaçăo dos 12 pacientes estu iados estão referidos na tabela 1. Destes pacientes, 6 foram internados na Enfermaria da Clínica Neurológica do Hospital das Clínicas da Faculdade de Medicina da. Universidade de Săo Paulo e um foi acom-

Trabalho da Clínica Neurológica (Prof. Horácio M. Canelas) do Departamento de Neuropsiquiatria da Faculdade de Medicina da Universidade de Sáo Paulo: *Médico adido; *Docente Livre. 


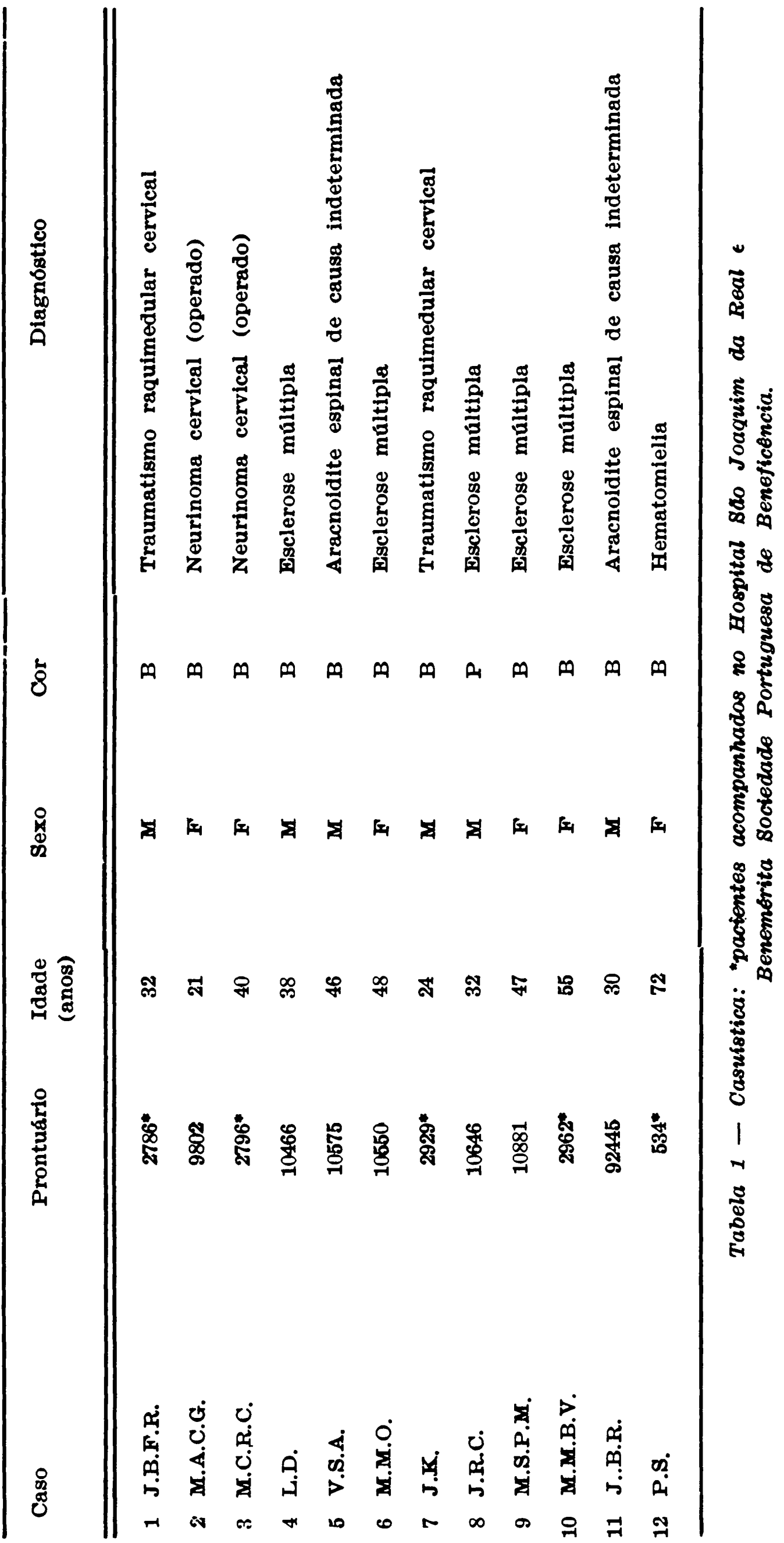


panhado no Ambulatório do mesmo Hospital; os 5 restantes foram acompanhados no Ambulatório do Hospital Săo Joaquim da Real e Benemérita Sociedade Portuguêsa de Beneficencia de Săo Paulo. As idades variaram de 21 a 72 anos (média de 40 anos); 6 pacientes eram do sexo masculino e 6 do sexo feminino, sendo que um era de cor preta e 11 de cor branca.

Quanto ao diagnóstico, em 5 pacientes a espasticidade era devida a esclerose múltipla com sintomatologia predominantemente medular, em 2 a traumatismo raquimedular cervical, em 2 a aracnoidite espinal de causa indeterminada, em 2 a neurinoma cervical operado e, em 1, a hematomielia (Tabela 1).

A tabela 2 refere-se ao método de avaliação utilizado, isto é, à quantificação clínica da sintomatologia neurologica (Alexander 1, modificado por Canelas 5). Os autores destacaram a sintomatologia da espasticidade (hipertonia, reflexos, clono e automatismos), além de estudarem a motricidade voluntária. Para cada um dos sintomas foi dado um valor que se encontra à direita da tabela. A soma destes valores dava o cômputo para cada avaliaçăo, que foi feita antes, durante e apos o tratamento, que durou entre 15 e 79 dias (mé̉ia 45 dias). Mediante a análíse dos cômputos foi possível comparar o que estava ocorrendo durante a evolução e tírar conclusర̃es.

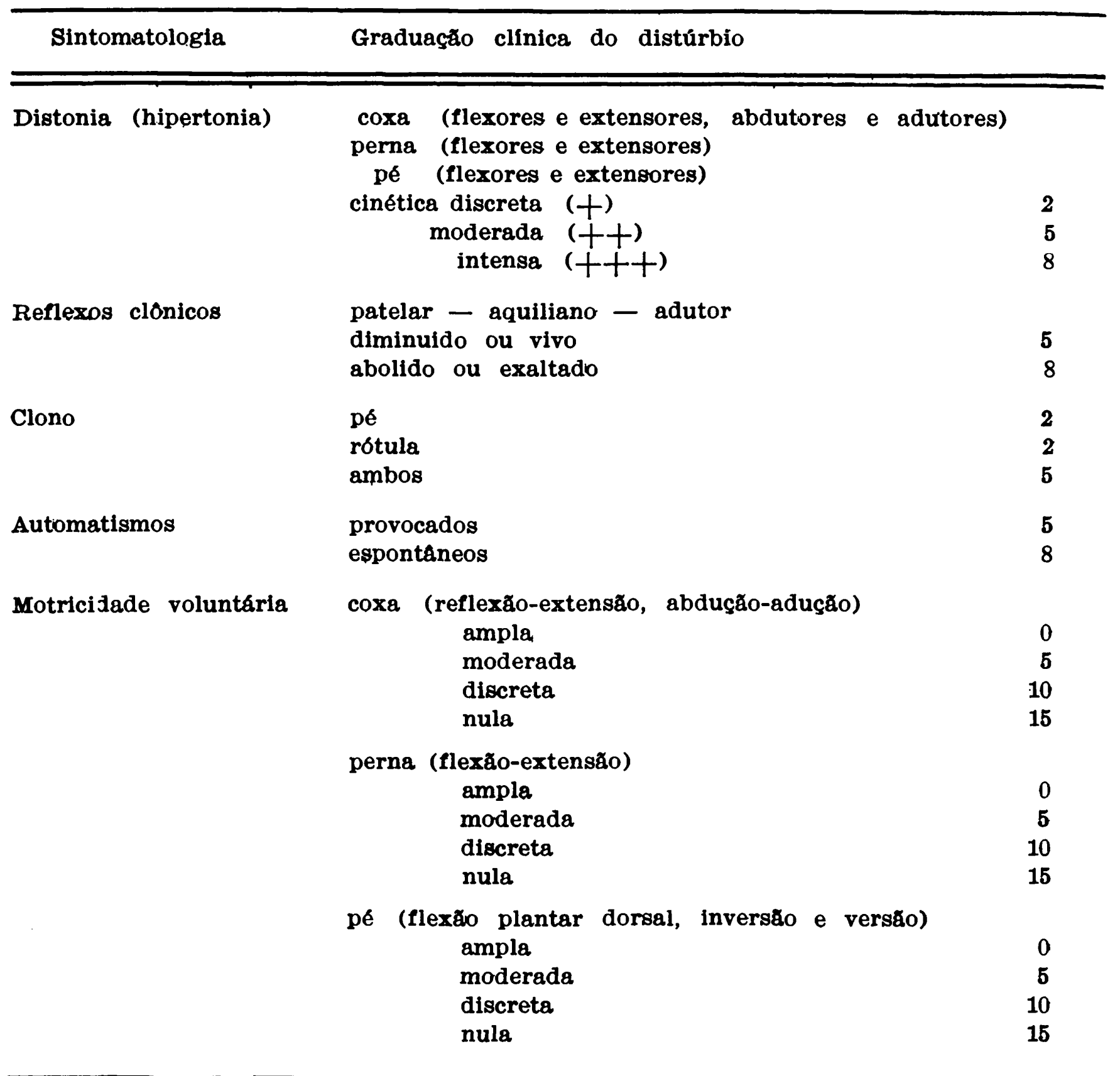

Tabela \& - Tabela de quantificaça clinica da sintomatologia neurologica nos membros inferiores, devendo os dados serem pesquisados d direita $e$ d esquerda. 
Foram realizados exames neurologicos antes do tratamento e após início do mesmo, com reexames semanais até completar 4 semanas de observaçăo. Os casos $2,4,7$ e 9 ficaram em observaçăo durante um periodo menor, que variou de 15 a 26 dias.

O tempo decorrido entre o inicio da incapacidade e a data do primeiro exame variou de 4 a 408 meses.

O tempo entre o início do tratamento e a alta variou de 15 a 79 dias, com a média de 45 dias (Tabela 3 ).

As doses totais de Ciba 34.647-Ba* utilizadas variaram de 300 a $5860 \mathrm{mg}$. A dose diária variou de 15 a $100 \mathrm{mg}$, com a média de $30 \mathrm{mg}$ (Tabela 3).

\section{RESULTADOS}

Quanto ao total dos cômputos estudados no início e por ocasiăo da alta verificamos que em dois casos năo houve qualquer modificaçăo, enquanto nos demais observamos (Tabela 3): a) diminuiçăo dos cômputos totais tanto no membro inferior díreito (MID) como no inferior esquerdo (MIE) em 8 casos; b) diminuíçăo dos cômputos totais em MID em 2 casos; c) năo se observou em qualquer caso diminuiçăo dos cômputos totais somente no MIE.

Em 5 casos, com o aumento da dose até 40 ou $50 \mathrm{mg}$ diariamente, ocorreu acentuada diminuiçăo da hipertonia a ponto de incapacitar a deambulaçă em 1 caso ocorreram também sensaçōes parestésicas difusas pelo corpo (Tabela 3); este efeito diminuíu com o abaixamento das doses do medicamento.

Foram analisados os resultados obtidos em relaçăo a cada parametro estudado (Tabela 4).

Quanto a hipertonia: a) diminuiçăo dos cômputos tanto em MID como MIE em 5 casos; b) diminuiçăo somente no MID em 2 casos; c) diminuiçăo somente no MIE em 2 casos; d) inalterados, 3 casos.

Com relaçăo aos reflexos profundos: a) díminuiçăo dos cômputos tanto em MID como MIE em 5 casos; b) diminuiçăo somente no MID em 1 caso; c) diminuiçăo somente no MIE em nenhum caso; $d$ ) inalterados, 6 casos.

O clono teve o seguinte comportamento: a) o caso 2 năo apresentava clono; b) diminuiçăo dos cômputos tanto em MID como MIE em 3 casos; c) díminuiçăo somente no MID em 2 casos; d) diminuição somente no MIE, nenhum caso; e) inalterados, 6 casos.

Os automatismos tiveram a seguinte evoluçăo: a) nåo apresentavam automatismos 3 casos; diminuiçấo dos cômputos tanto em MID como MIE em 6 casos; b) diminuição somente no MID ou no MIE em nenhum caso; c) inalterados, 3 casos.

Com relaçåo à motricidade voluntária: a) um caso não apresentava alteraçóes da motricidade voluntária; b) dimiuiçăo dos cômputos tanto em MID como MIE em 4 casos; c) diminuição somente no MID em nenhum caso; d) diminuiçăo somente no MIE em 1 caso; e) inalterados, 6 casos.

\section{COMENTARIOS E CONCLUSOES}

$\mathrm{Na}$ última década foram realizados estudos experimentais com Ciba 34.647-Ba em animais de laboratório que nem sempre se prestam à comparação com o que ocorre na espasticidade humana. As observações até agora realizadas por outros investigadores são heterogêneas não só quanto ao métodos de avaliação empregados (eletromiografia, reflexo $\mathrm{H}$, avaliações quantitativas relacionadas

"Lioresal, CIBA-GEIGY Química S.A. 


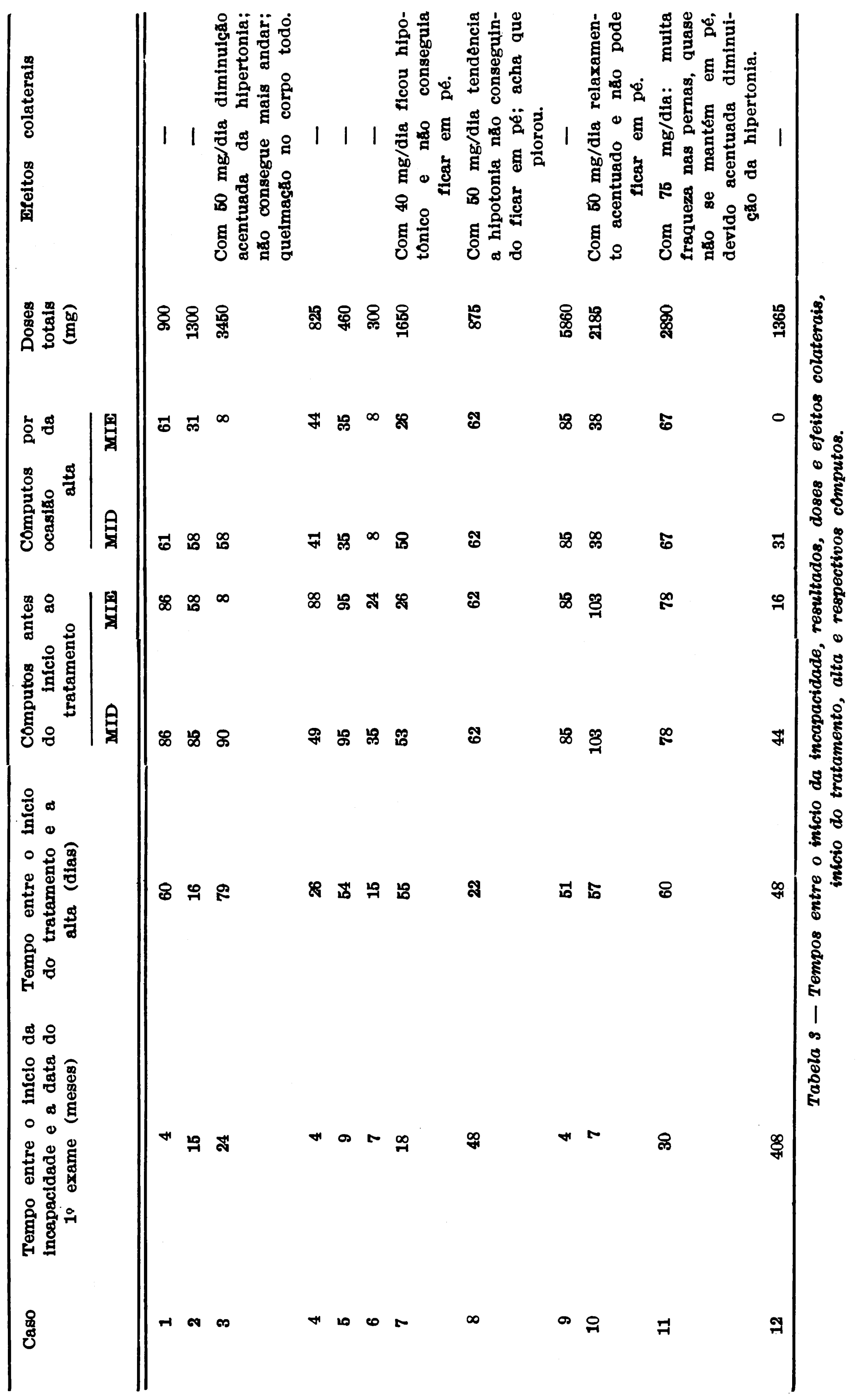




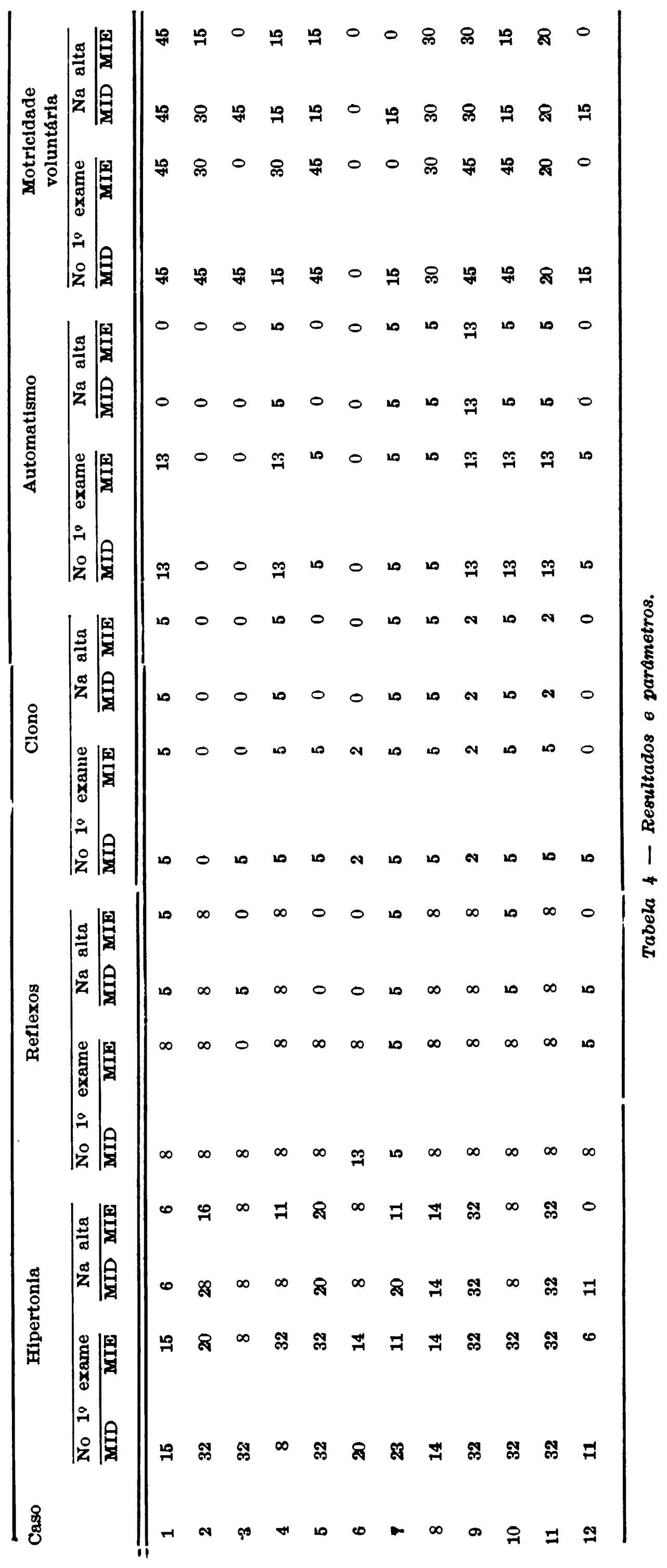


com atividade de vida diária) como também em relação à heterogeneidade do material por reunirem em um mesmo grupo mielopatias e encefalopatias. Entretanto, todos eles concluem que o medicamento atua na espasticidade humana, promovendo diminuição ou abolição da mesma.

No presente trabalho foi utilizado, em 12 pacientes, critério exclusivamente clínico e de fácil aplicação, bem como material até certo ponto heterogêneo mas com lesões predominantemente medulares.

Os resultados obtidos demonstram que a droga diminui a hipertonia em cerca de $75 \%$ dos casos; em $50 \%$ dos casos, os reflexos que eram exaltados passaram a vivos ou normalizaram; em cerca de $70 \%$ dos casos diminuiram os automatismos e as trepidações epileptóides.

Embora os parâmetros relativos à espasticidade clínica tenham diminuido ou desaparecido em cerca de $60 \%$ dos casos, a melhora relativa à motricidade voluntária e conseqüentemente à função motora foi pequena, em virtude da persistência da hipertonia cinética, sobre a qual a droga não se mostrou tão eficiente.

Por outro lado, em nenhum dos 12 pacientes ocorreram manifestações de intolerância ao medicamento, mesmo considerando que um dos pacientes recebeu dose superior a $100 \mathrm{mg}$ diários.

De acordo com estas observações pode-se concluir que o Ciba 34.647-Ba reduz a espasticidade clínica sem, entretanto, agir na hipertonia cinética, o que não invalida seu emprego em casos específicos, isto é, naqueles pacientes com hipertonia cinética discreta ou nula.

Acreditamos (e isto será objeto de futuras investigações) que a associação do Ciba 34.647-Ba com um derivado diazepínico possa resultar em diminuição da espasticidade clínica e da hipertonia cinética.

\section{RESUMO}

Utilizando a quantificação clínica da sintomatologia neurológica os autores fizeram observações sobre o Ciba 34.647-Ba e avaliaram pacientes com espasticidade e incapacidade motora devidas a lesões da medula espinal. São relatados us resultados, salientando os efeitos do medicamento sobre os principais componentes da espasticidade (hipertonia, hiperreflexia, clono e automatismos). As teorias propostas para explicar o mecanismo de ação da droga são mencionadas. São também referidas as doses utiizadas, duração da ação músculo- relaxante, tolerância e efeitos colaterais.

SUMMARY

\section{Clinical quantification of the action of CIBA 34.647 BA on spasticity}

Using the clinical quantification of the neurologic symptomatology the authors made observations and avaliated the action of Ciba 34.647- $\mathrm{Ba}$, a GABA derivative, in 12 patients suffering from spasticity and motor incapacity due to 
spinal cord lesions. The results are reported emphasizing the effects of the drug on the main components of spasticity (hypertonus, hyperactivity of the deep reflexes, clonus and automatisms). Some theories explaining the mechanism of action of the drug are mencioned. References are made about doses, duration of action, tolerance and side-effects.

\section{REFHERENCIAS}

1. ALEXANDER, L. - New concept of critical steps in course of chronic debilitating neurologic disease in evaluation of therapeutic response. Arch. Neurol. Psychiat. (Chicago) $66: 253,1951$.

2. ASHBY, P. \& WHITE, D. G. - Presynaptic inhibition in spsticity and the effect of beta (4-chlorophenyl) GABA. J. Neurol. Sci. 20:329, 1973.

3. BERGAMINI, L.; RICCIO, A. \& BERGAMASCO, B. - Un farmaco ad azione antispastica della muscolatua striata: sperimentazione clinica di un derivato del GABA. Minerva Med. 57:2723, 1966.

4. BURKE, D.; ANDREWS, C. J. \& KNOWLES, L. - The action of a GABA derivative in human spsticity. J.Neurol.Sci. 14:199, 1971.

5. CANELAS, H. M. - Quantificaçăo clínica da sintomatologia neurológica. Rev. paul. Med. 63:178, 1963.

6. CASTANEDA, C. - Investigacion de Ciba 34.647-BA en los sindromes espasticos neurologicos. Sem.méd.Méx. $54: 13,1967$.

7. Castaigne, P.; held, J. P.; LAPLANE, D.; PIERROT-Deseilligigy, E.; BUSEEL, B. \& MACQUART-MOULIN, J. - Ftude de l'effect du Lioresal dans la spesticité. Rev.Neurol. (Paris) 128:245, 1973.

8. DAVIDOFF, R. A. \& SEARS, E. S. - The effects of Lioresal on synaptic activity in the isolated spinal cord. Neurology (Minneapolis) 24:957, 1974.

9. FROM, A. \& HELTBERG, A. - A. double-blind trial with Baclofen (Lioresal) and Diazepam in spsticity due to multiple sclemsis. Acta Neurol. Scand. 51:158, 1975.

10. JONES, R. F.; BURKE, D.; MAROSSZEKY, J. E. \& GILLIES, J. D. - A new agent for the control of spasticity. J. Neurol. Neurosurg. Psychiat. 53:464, 1970.

11. KNUTSSON, E.; LINDBLOM, U. \&. MARTENSSON, A. - Differences in effects in gamma and alpha spasticity induced by the GABA derivative Baclofen (Lioresal). Brain 96:29, 1973.

12. MORALES, L. R. - Efectos clinicos y terapeuticos del Ciba 34:756-Ba en los sindromes espasticos. Mex. Med. 54:187, 1967.

13. MOROSINI, C. \& MARINO, A. - La terapia antispastica quale coadiuvante della rieducazione motoria nei neurolesi centrali: esperimentazione clinica con un derivato dell'acido amino butirico ad azione inibente sulle transmissioni poli e monosinaptiche midollari. Europa Medicophysica 4:37, 1968.

14. PEDERSEN, E.; ARLIEN-SOBORG, P.; GRYNDERUP, V. \& HENRIKSEN, O. - GABA derivative in spasticity. Acta Neurol. Scand. 46:257, 1970.

15. PEDERSEN, E.; ARLIEN-SOBORG, P. \& MAI, J. The mode of action of the GABA derivative Baclofen in human spasticity. Acta Neurol. Scand. 50:665, 1974.

16. PIERU, F. K. \& ZIMMERMAN, P. Action of a GABA-derivative on post-synaptic potentials and membrane properties of cats'spinal motoneurones. Brain Res. 54: $376,1973$.

Clinica Neurologica - Fraculdade de Medicina, Universidade de Sdo Paulo - Caixa Postal S\$61 - 01000 sao Paulo, SP - Brasil 